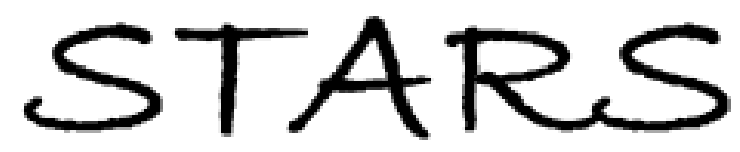

University of Central Florida

STARS

$1-1-2010$

\title{
Flow regime transition at high capillary numbers in a microfluidic T-junction: Viscosity contrast and geometry effect
}

Amit Gupta

Ranganathan Kumar

University of Central Florida

Find similar works at: https://stars.library.ucf.edu/facultybib2010

University of Central Florida Libraries http://library.ucf.edu

This Article is brought to you for free and open access by the Faculty Bibliography at STARS. It has been accepted for inclusion in Faculty Bibliography 2010 s by an authorized administrator of STARS. For more information, please contact STARS@ucf.edu.

\section{Recommended Citation}

Gupta, Amit and Kumar, Ranganathan, "Flow regime transition at high capillary numbers in a microfluidic T-junction: Viscosity contrast and geometry effect" (2010). Faculty Bibliography 2010s. 209.

https://stars.library.ucf.edu/facultybib2010/209

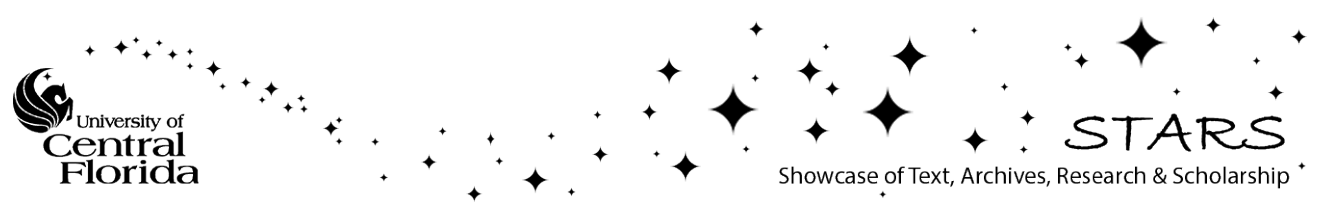




\section{Flow regime transition at high capillary numbers in a microfluidic T-junction: Viscosity contrast and geometry effect}

Amit Gupta, and Ranganathan Kumar

Citation: Physics of Fluids 22, 122001 (2010); doi: 10.1063/1.3523483

View online: https://doi.org/10.1063/1.3523483

View Table of Contents: http://aip.scitation.org/toc/phf/22/12

Published by the American Institute of Physics

\section{Articles you may be interested in}

Formation of dispersions using "flow focusing" in microchannels

Applied Physics Letters 82, 364 (2003); 10.1063/1.1537519

Droplet formation and stability of flows in a microfluidic T-junction

Applied Physics Letters 94, 164107 (2009); 10.1063/1.3116089

Droplet formation in a T-shaped microfluidic junction

Journal of Applied Physics 106, 034906 (2009); 10.1063/1.3187831

Droplet formation in microfluidic cross-junctions

Physics of Fluids 23, 082101 (2011); 10.1063/1.3615643

Capillary threads and viscous droplets in square microchannels

Physics of Fluids 20, 053302 (2008); 10.1063/1.2911716

Droplet breakup in microfluidic T-junctions at small capillary numbers

Physics of Fluids 21, 072001 (2009); 10.1063/1.3170983

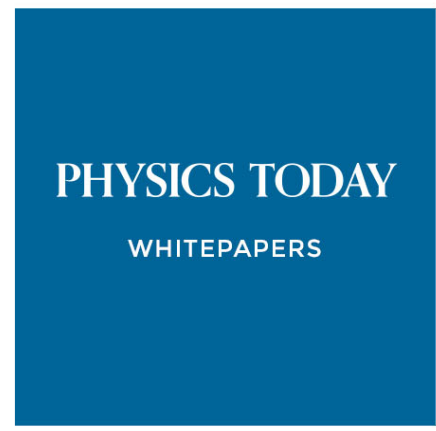




\title{
Flow regime transition at high capillary numbers in a microfluidic T-junction: Viscosity contrast and geometry effect
}

\author{
Amit Gupta ${ }^{1, a)}$ and Ranganathan Kumar, ${ }^{2, b)}$ \\ ${ }^{1}$ Department of Aerospace Engineering, University of Michigan, Ann Arbor, Michigan 48109, USA \\ ${ }^{2}$ Department of Mechanical, Materials and Aerospace Engineering, University of Central Florida, \\ Orlando, Florida 32816, USA
}

(Received 3 January 2010; accepted 10 November 2010; published online 2 December 2010)

\begin{abstract}
Flow regimes obtained as a consequence of two immiscible fluids interacting at a T-junction are presented for transitional to high capillary numbers and different ratios of the continuous and dispersed phase flow rates and viscosities. Results are presented for the formation of micron-sized droplets using simulations performed based on a three-dimensional lattice Boltzmann method. The influence of viscosity and geometry of the device on the frequency and volume of droplets formed has been examined and the nondimensional drop size correlated with the capillary number and flow rate ratio. This work reveals two important and new physical features: (a) the transition zone separating droplet and jet flows narrows for high capillary numbers and (b) the critical flow rate ratio separating droplet and parallel flows increases as the dispersed to continuous channel width ratio increases, aspects which have been correlated using a simple relation for both transitions from the droplet-at-T-junction to droplet-in-channel and droplet-in-channel to parallel flow. In the droplet-at-T-junction regime, the droplet formation frequency was recorded as a function of the capillary number, flow rate ratio, and the channel width ratio as well. Results show that the transition to stable jets can be delayed and droplets can be formed even at very high flow rate ratios by significantly increasing the viscosity of the continuous phase. (C) 2010 American Institute of Physics. [doi:10.1063/1.3523483]
\end{abstract}

\section{INTRODUCTION}

Microfluidic devices that generate emulsions of liquid droplets suspended in an outer immiscible liquid could potentially have widespread applications in food processing, drug delivery, microanalysis, tumor destruction, as ultrasound agents, and in chemical reactions at the micron level. ${ }^{1,2}$ Many applications are found that involve multiphase transport through thin capillaries and microchannels. For instance, miniature evaporative and distillation units and bubble generators, flow cytometers, and emulsions all use transport of two immiscible fluids through a microchannel. Over the past few years, numerous experiments have highlighted the advances being made in the field of droplet generation using a variety of microfluidic devices, particularly using coflowing fluids, ${ }^{3,4}$ passing immiscible fluids through an orifice, ${ }^{5-7}$ and droplet formation at T-junctions. ${ }^{8-19}$ Due to the length scales involved, the flow of the immiscible fluids falls in the laminar regime, which enables a higher degree of control on the injection process. $^{2}$ The large surface-tovolume ratio of the microchannel enhances the role of surface tension effects as well. The other aspects that influence such a class of problems are the flow rates of the continuous and dispersed phases, viscosities of the fluids, geometry of the device, and interplay of surface tension and viscous forces.

The past decade has seen a steady increase in experi-

\footnotetext{
a)Electronic mail: amitgupt@umich.edu.

${ }^{\text {b) }}$ Author to whom correspondence should be addressed. Electronic mail: rnkumar@mail.ucf.edu.
}

mentation on the formation of droplets in T-junction devices. Experiments have shown the applicability of such a device to produce droplets that could generate ordered or complex patterns based on the flow rates of the two liquids, ${ }^{8}$ namely, oil and water. A similar design has been shown to yield equal and periodic distribution of droplets that could be collected at the two outlets of a T-junction. ${ }^{15}$ The formation of droplets and plugs has also been accomplished in a T-junction microfluidic device using both high and low viscous aqueous solutions and the mixing of these reagents could be enhanced by moving droplets through curving microchannels. ${ }^{11}$

$\mathrm{Xu}$ et al. ${ }^{16}$ have shown the formation of air microbubbles surrounded by an aqueous phase that was accomplished with less than $2 \%$ polydispersity in sizes of the bubbles produced. They show that increasing the continuous phase viscosity at a fixed flow rate of the dispersed and continuous fluids results in lower volumes of the droplets formed at the T-junction, a result that is consistent with other experimental observations. In a separate study, it was shown that the volume of the droplets formed decreases rapidly as the continuous flow rate is increased at a constant flow rate of the dispersed phase. ${ }^{17}$ These recorded droplet volumes were also found to be a function of the viscosity of the continuous phase. Engl et al. ${ }^{18}$ report that a train of confined droplets flowing through a T-junction channel can be distributed or collected into the shortest channel for low capillary number flows. The effect of temperature on the droplet formation mechanism in a $\mathrm{T}$-junction microchannel has also been examined using $\mathrm{TiO}_{2}$ nanoparticles of size $15 \mathrm{~nm}$ dispersed in water as the dispersed phase in a channel with 
width ratio $\Lambda=w_{d} / w_{c}=1 / 3,{ }^{19}$ where $w_{d}$ and $w_{c}$ are the widths of the dispersed and continuous channels, respectively. As the surface tension and viscosities inherently reduce with an increase in temperature of the system, their findings reveal that droplets of a relatively larger size are formed as compared to droplets formed by using de-ionized water as the dispersed phase at the same temperature.

Based on experimental observations, various correlations have been proposed to quantify the droplet sizes formed at a T-junction. Garstecki et al., ${ }^{9}$ who studied droplet formation for low capillary number flows and for channels where $\Lambda=w_{d} / w_{c}$ lies between 0.25 and 1 , proposed that the lengths of the drops can be written as

$$
L / w_{c}=1+\alpha Q,
$$

where

$$
\alpha=d / w_{c}
$$

is a fitting parameter based on the characteristic width of the neck $(d)$, which depends on the geometry of the device, and $Q=Q_{d} / Q_{c}$ is the ratio of the flow rates of the dispersed $\left(Q_{d}\right)$ and continuous $\left(Q_{c}\right)$ liquid. However, the prediction of this fitting parameter was difficult, as the speed at which the neck collapses on the sharper downstream edge of the dispersed phase channel and the speed of the continuous fluid are not the same. ${ }^{9}$ Although Eq. (1) was found to match well with the experimental results, the authors noticed that the applicability is questionable for channel geometries, where $\Lambda=w_{d} / w_{c}<1 / 2$. On the other hand, the formation of droplets has been proposed to be a competition between the interfacial force resisting the detachment of the expanding droplet and the combined force of the viscous drag (acting due to the flow of the continuous phase) and the pressure force (generated due to a blockage of the passage for the continuous phase to flow because of the protruding dispersed phase). ${ }^{14}$ The interfacial, shear, and pressure forces in the original work were written as

$$
\begin{aligned}
& F_{\text {stension }} \approx \sigma h, \\
& F_{\text {shear }} \approx \frac{\mu_{c} Q_{c} b}{\left(w_{c}-b\right)^{2}}, \\
& F_{p} \approx \frac{\mu_{c} Q_{c} b^{2}}{\left(w_{c}-b\right)^{3}},
\end{aligned}
$$

where $\sigma$ is the interfacial tension parameter, $h$ is the depth of the assembly, $\mu_{c}$ is the dynamic viscosity of the continuous phase, and $b$ is the length of the droplet before pinch-off. Following a force balance $F_{\text {stension }}=F_{\text {shear }}+F_{p}$, one can get

$$
(1-\bar{b})^{3}=\bar{b} \mathrm{Ca},
$$

where $\bar{b}$ is the normalized length of the droplet before pinchoff and $\mathrm{Ca}=\mu_{c} Q_{c} / w_{c} h \sigma$ is the capillary number. The roots of the cubic polynomial were used to estimate the dimensionless volume of the droplet formed as

$$
\bar{V}=\frac{V}{w_{c}^{2} h} \approx \bar{b}^{2}+\Lambda Q .
$$

The experimental data, when compared with Eq. (7), were found to be in reasonable agreement. However, due to an approximate form of the forces and simplistic assumptions that were made to predict the dynamics of droplet formation in this model, discrepancies could arise when comparing other experimental data with Eq. (7). ${ }^{14}$

Stability of parallel flows in a microfluidic T-junction has also received attention. ${ }^{12}$ Based on the flow rates of the continuous and dispersed liquid, a regime map was created that distinguished three modes of flow encountered in T-junctions: droplets formed at the T-junction (DTJ), droplets formed in the channel (DC), and parallel flow (PF). In addition to the flow rates, the stability of parallel flows in a funneled T-junction could also be linked to the wetting behavior, viscosity contrast, and aspect ratio of the inlet channel for the two liquids. ${ }^{12}$ Since the existing analyses do not take these flow regimes into consideration, a more robust and unified model is required that can predict the possible outcomes for the T-junction microchannel.

In spite of the rapid advancements in the past few years in the fabrication and examination of T-junction devices, theoretical work on exploring the interplay of surface tension and viscous forces has received limited attention, ${ }^{10,20-22}$ partly due to the complex nonlinear behavior that emanates as a consequence of two laminar streams interacting in a micron-sized channel. Using free-energy based lattice Boltzmann simulations, for $\Lambda=1,0.0033 \leq \mathrm{Ca} \leq 0.065$, $1 / 20 \leq Q \leq 1$, and a fixed ratio of the dispersed to continuous phase viscosity ratio $\lambda=\mu_{d} / \mu_{c}=3.44$, where $\mu_{d}$ is the dynamic viscosity of the former, it has been proposed that the radius of droplets $(r)$ at pinch-off follows a relationship given by ${ }^{10}$

$$
\frac{r}{w_{c}} \propto \mathrm{Ca}^{-1 / 4} .
$$

On the other hand, a separate study has taken the viscosity contrast into consideration by simulating droplet formation for viscosity ratios in the range $1 / 8 \leq \lambda \leq 1 .{ }^{20}$ However, the widths of the dispersed and continuous liquid channels were equal (i.e., $\Lambda=1$ ) and the flow rate ratio was limited to $Q=1 / 8$ and $1 / 4$. Thus, the simulations reported in both these studies have focused primarily on viscosity ratios $\lambda=\mu_{d} / \mu_{c}$ $>1 / 8$ and aspect ratios $w_{c} / h=1$ and $\Lambda=w_{d} / w_{c}=1$. In a recent study, ${ }^{21}$ we analyzed the flow regimes in a T-junction for high capillary numbers and different flow rates of the continuous and dispersed phases. Our simulations, conducted for a single geometry $\left(\Lambda=w_{d} / w_{c}=0.5\right.$ and $\left.h / w_{c}=0.5\right)$, show that the transition zone, which separates parallel and droplet flows, shrinks at high capillary numbers. Clearly, an investigation into the role of the device geometry (i.e., for a wide range of $\Lambda$ ) and contrasting viscosities of the two liquids has not been carried out in the dripping regime, where squeezing pressure is not the dominant mechanism for droplet formation. 
In summary, experiments ${ }^{8-10,13,14,16}$ have resulted in different models for predicting the droplet size for different flow conditions, which have only been applicable for a certain set of experimental data. As a result, at times, the predictions can be incongruous. In addition, no theoretical study exists that has investigated the effect of the geometry of the microfluidic device and large viscosity contrast on the size and shape of the droplets collected downstream at the outlet. A comprehensive modeling effort is required to analyze the flow at a T-junction, which could lead to an understanding of the nature of the forces and predict the influence of the channel geometry and fluid properties on the operating flow rates that yield droplets of a certain size and frequency. Through lattice Boltzmann simulations, we aim to establish the effect of fluid properties and device configuration in the (stable) droplet formation regime.

Hence, the objective of this work is to (a) simulate and classify the droplets formation process in T-junction microfluidic devices, (b) study the role of the geometry of the device to attain steady formation of droplets, and (c) quantify the frequency of droplet formation for a range of the channel widths given by $1 / 5 \leq \Lambda \leq 1 / 2$ and viscosity ratios $1 / 100$ $\leq \lambda \leq 1 / 10$ for high capillary number flows. This is the first study to examine the effect of these factors on the frequency of droplet formation for a range of flow rates, channel widths, and very small viscosity ratios.

\section{METHODOLOGY}

The lattice Boltzmann method (LBM) can be regarded to be a finite-difference formulation for the kinetic equation of the particle distribution function $\left(f_{i}\right)$ along the direction " $i$ ",23 and is given as

$$
\begin{aligned}
& f_{i}\left(\mathbf{x}+\mathbf{e}_{i} \delta t, t+\delta t\right)=f_{i}(\mathbf{x}, t)+\Omega_{i}\left[f_{i}(\mathbf{x}, t)\right], \quad i=0,1, \ldots, N, \\
& \text { where } \Omega_{i}\left[f_{i}(\mathbf{x}, t)\right]=-\frac{f_{i}(\mathbf{x}, t)-f_{i}^{\mathrm{eq}}(\mathbf{x}, t)}{\tau} .
\end{aligned}
$$

$\mathbf{e}_{i}$ is the lattice speed of particles moving in direction $i . N$ denotes the number of links at each lattice point. $\Omega_{i}$ is the linearized collision operator with the single relaxation time approximation and is commonly known as the lattice Bhatnagar-Gross-Krook (BGK) operator. $f_{i}^{\text {eq }}$ and $\tau$ are the local equilibrium distribution and the dimensionless relaxation time, respectively. The macroscopic density and momentum flux can be obtained from the distribution functions by

$$
\rho=\sum_{i} f_{i}=\sum_{i} f_{i}^{\mathrm{eq}} ; \quad \rho \mathbf{u}=\sum_{i} f_{i} \mathbf{e}_{i}=\sum_{i} f_{i}^{\mathrm{eq}} \mathbf{e}_{i},
$$

where

$$
f_{i}^{\mathrm{eq}}=\gamma_{i} \rho\left[1+3 \mathbf{e}_{i} \cdot \mathbf{u}+\frac{9}{2}\left(\mathbf{e}_{i} \cdot \mathbf{u}\right)^{2}-\frac{3}{2} \mathbf{u} \cdot \mathbf{u}\right]
$$

and for the D3Q19 lattice used in the current study, the weights $\gamma_{i}$ are given as

$$
\gamma_{i}=\left\{\begin{array}{l}
1 / 3 \text { for } i=0 \\
1 / 18 \text { for } i=1, \ldots, 6 \\
1 / 36 \text { for } i=7, \ldots, 18
\end{array} .\right.
$$

Chen and Doolen ${ }^{23}$ have shown that Eq. (9) can be reduced to the Navier-Stokes equations in the low frequency, long wavelength limit using the Chapman-Enskog expansion together with Eq. (10). As a result, the pressure and kinematic viscosity are written as $p=c_{s}^{2} \rho$ and $\nu=(\tau-1 / 2) c_{s}^{2} \delta t$, respectively.

Numerous methods have been used by researchers over the past decade to conduct multiphase simulations using LBM. These include the chromodynamic, ${ }^{24}$ pseudopotential, ${ }^{25}$ free-energy, ${ }^{26}$ and the projection ${ }^{27}$ methods. Each of these formulations has its own advantages and disadvantages. The chromodynamic and pseudopotential methods are widely used to simulate multiphase flows because of their ease of implementation and isotropic nature of solutions. However, (a) velocity vectors are high at the interface, (b) momentum is not conserved locally, and (c) the models work well for low density ratios only. On the other hand, in the "free-energy" approach, local momentum conservation was satisfied, although the model suffers from the lack of Galilean invariance. In a recent and robust scheme for the treatment of the interface, ${ }^{28}$ a two-fluid mixture is kept separated through a diffuse interface that spans a few lattice nodes. The location of the interface is controlled by a surface tension force which is incorporated such that the stress boundary condition and the continuity of velocity across the interfacial line are satisfied.

This model begins with an elementary two-phase method $^{24}$ in which two particle distributions denote the two different fluids. Each fluid is assigned a "color" (the use of the word color is meant for identifying the fluid only) at all locations in the domain of interest. Each of these colored phases undergoes the collision and streaming operations under the incompressible ${ }^{29}$ BGK approximation, given as

$$
f_{i}^{k}\left(\mathbf{x}+\mathbf{e}_{i} \Delta t, t+\Delta t\right)=f_{i}^{k}(\mathbf{x}, t)-\frac{f_{i}^{k}(\mathbf{x}, t)-f_{i}^{k, \mathrm{eq}}(\mathbf{x}, t)}{\tau^{k}}+\Phi_{i},
$$

where $k$ denotes the "red" (R) or "blue" (B) distribution and

$$
f_{i}^{k, \text { eq }} \quad \begin{array}{ll}
\frac{1}{3}\left(\rho_{k}-\frac{\mathbf{u}_{k} \cdot \mathbf{u}_{k}}{2 c_{s}^{2}}\right), & i=0 \\
\frac{1}{18}\left(\rho_{k}+\frac{\mathbf{e}_{i} \cdot \mathbf{u}_{k}}{c_{s}^{2}}+\frac{\left(\mathbf{e}_{i} \cdot \mathbf{u}_{k}\right)^{2}}{2 c_{s}^{4}}-\frac{\mathbf{u}_{k} \cdot \mathbf{u}_{k}}{2 c_{s}^{2}}\right), & 1 \leq i \leq 6 \\
\frac{1}{36}\left(\rho_{k}+\frac{\mathbf{e}_{i} \cdot \mathbf{u}_{k}}{c_{s}^{2}}+\frac{\left(\mathbf{e}_{i} \cdot \mathbf{u}_{k}\right)^{2}}{2 c_{s}^{4}}-\frac{\mathbf{u}_{k} \cdot \mathbf{u}_{k}}{2 c_{s}^{2}}\right), & 7 \leq i \leq 18
\end{array}
$$

for the D3Q19 lattice used in this study (see Fig. 1), with $c_{s}^{2}=1 / 3$ as the speed of sound in lattice units. More so, the density and velocity of each of the phases are given by

$$
\sum_{i} f_{i}^{k}(\mathbf{x}, t)=\rho_{k}=\frac{p_{k}}{\rho_{0} c_{s}^{2}},
$$




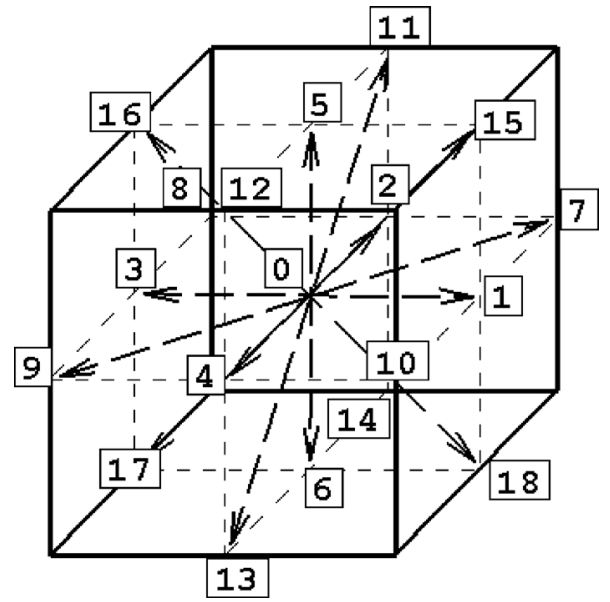

FIG. 1. Discrete velocity vectors for the 3-D 19 speed model.

$$
\sum_{i} f_{i}^{k} \mathbf{e}_{i}=\mathbf{u}_{k},
$$

where $\rho_{0}$ is the density of the fluid and is a constant parameter. This formulation of the LBE recovers the steady incompressible Navier-Stokes equation.

The original model ${ }^{24}$ suffered from the side effect of introducing anisotropy and high spurious velocities at the interface, which were relieved in the recent formulation by the introduction of a direct forcing term at the sites where the mixing of the two phases occurs. In order to impose the stress boundary condition and the continuity equation for the incompressible fluids, Lishchuk's model ${ }^{28}$ incorporated a local pressure gradient throughout the interface. This pressure force is defined such that it acts normal to the interface, centripetally and with a magnitude that is proportional to the gradient of the phase field $\rho^{N}$, defined as

$$
\rho^{N}(\mathbf{x}, t)=\left[\frac{\rho_{R}(\mathbf{x}, t)-\rho_{B}(\mathbf{x}, t)}{\rho_{R}(\mathbf{x}, t)+\rho_{B}(\mathbf{x}, t)}\right] .
$$

Clearly, $-1 \leq \rho^{N}(\mathbf{x}, t) \leq 1$. The local curvature at the "mixed" locations on the interface is computed based on the normal vector computed at the particular lattice location. The normal vector $\mathbf{n}$ is defined as a function of the phase field and is given by

$$
\mathbf{n}=-\frac{\nabla \rho^{N}}{\left|\nabla \rho^{N}\right|} .
$$

Based on the local normal vector, the radius of curvature $R$ of the interface is computed as

$$
\kappa=\frac{1}{R}=-\nabla_{S} \cdot \mathbf{n},
$$

where $\kappa$ is the curvature, and

$$
\nabla_{S}=(\mathbf{I}-\mathbf{n n}) \cdot \nabla,
$$

where $\mathbf{I}$ is the unit tensor and $\nabla_{S}$ is the surface gradient operator.

Based on the curvature, the source term $\Phi_{i}$ is calculated such that it incorporates the surface tension force and is given as

$$
\Phi_{i}=\frac{\gamma_{i}}{c_{s}^{2}} \mathbf{F} \cdot \mathbf{e}_{i},
$$

where

$$
\mathbf{F}(\mathbf{x}, t)=-\frac{1}{2 R} \sigma \nabla \rho^{N} .
$$

The partial derivatives of the components of the normal vector are computed based on a formulation for discretizing a general function $\phi$ on a regular lattice with contributions from the neighboring lattice sides

$$
\frac{\partial \phi}{\partial x}=\frac{1}{c_{s}^{2}} \sum_{i} \phi\left(\mathbf{x}+\mathbf{e}_{i}\right) e_{i x} \gamma_{i}
$$

and

$$
\frac{\partial \phi}{\partial y}=\frac{1}{c_{s}^{2}} \sum_{i} \phi\left(\mathbf{x}+\mathbf{e}_{i}\right) e_{i y} \gamma_{i} .
$$

In addition, the color segregation step of the original model $^{24}$ was replaced by a recent variant, ${ }^{30}$ which minimizes the spurious velocities even further and at the same time removes lattice pinning. According to this method, the postcollision distributions of the two color fluids, red and blue, are computed as

$$
\begin{aligned}
& f_{i}^{R}=\frac{\rho_{R}}{\rho_{R}+\rho_{B}} f_{i}+\gamma_{i} \beta \frac{\rho_{R} \rho_{B}}{\left(\rho_{R}+\rho_{B}\right)} \cos \theta_{f}\left|\mathbf{e}_{i}\right|, \\
& f_{i}^{B}=\frac{\rho_{B}}{\rho_{R}+\rho_{B}} f_{i}-\gamma_{i} \beta \frac{\rho_{R} \rho_{B}}{\left(\rho_{R}+\rho_{B}\right)} \cos \theta_{f}\left|\mathbf{e}_{i}\right|,
\end{aligned}
$$

where

$$
\cos \theta_{f}=\frac{\mathbf{e}_{i} \cdot \nabla \rho^{N}}{\left|\mathbf{e}_{i}\right|\left|\nabla \rho^{N}\right|}
$$

and $\beta$ is the antidiffusion parameter and is fixed at 0.7 to keep the spurious currents low and maintain a narrow interface thickness. $^{22}$

\section{RESULTS AND DISCUSSION}

The important parameters that can influence the droplet formation process can be written in terms of the physical parameters that characterize the two-fluid flow and can be used to define six independent dimensionless parameters: ${ }^{20} Q=U_{d} w_{d} h / U_{c} w_{c} h=U_{d} w_{d} / U_{c} w_{c}, \mathrm{Ca}=\mu_{c} U_{c} / \sigma$, $\operatorname{Re}_{c}=\rho U_{c} w_{c} / \mu_{c}, \lambda=\mu_{d} / \mu_{c}, \Gamma=h / w_{c}$, and $\Lambda=w_{d} / w_{c}$, where $U_{c}$ and $U_{d}$ are the average velocities of the continuous and dispersed phase, respectively. The geometric parameters and the flow configuration for the three-dimensional geometry of the T-junction is shown in Fig. 2, where the top and side views depict the channel dimensions and the flow inlets for the continuous and dispersed phase to enter. For simplicity, the two liquids have been assumed to have equal density, given by $\rho_{0}=1$. No-slip boundary conditions were employed using the second-order accurate half-way bounce back scheme for the solid walls. ${ }^{23}$ The dispersed phase is nonwetting and the continuous phase wets the solid wall in the main channel. At the inlet and outlet, mass flux was prescribed at 


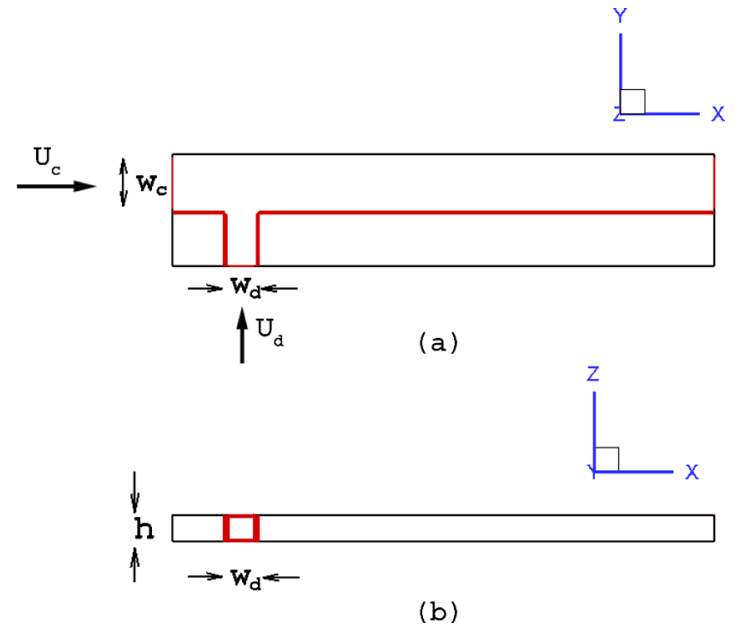

FIG. 2. (Color online) (a) Top and (b) side view of the T-junction microchannel used for modeling formation of droplets. The inlet channels are marked using velocities at the appropriate faces.

each time increment using $f_{i}^{\text {eq }}\left(\rho, \mathbf{u}_{\text {inlet }}\right){ }^{31}$ In addition, a constant outlet pressure was enforced at the exit of the continuous phase channel and the pressure at the inlet was allowed to develop to a steady-state value. ${ }^{32}$ Since the droplet formation process in microchannels is laminar in nature, the inertial forces are smaller or comparable to viscous forces. The multiphase model chosen in the current study guarantees low spurious velocities and numerical stability even for $\operatorname{Re}_{c} \ll 1.22$

The current multiphase model was validated by comparing the normalized droplet volume recorded using lattice Boltzmann simulations for $\Lambda=\Gamma=1$ and $\lambda=1 / 8$ at two different flow rate ratios of $Q=1 / 8$ and $1 / 4$ with the simulation results of the phase-field model ${ }^{20}$ and the predictions of the force balance given by Eq. (7). ${ }^{14}$ The mesh selected consisted of $201 \times 41 \times 21$ Cartesian equidistant nodes and the volume comparison is shown in Fig. 3. The current simulations were found to be in good agreement with the phasefield simulations, ${ }^{20}$ whereas deviation from the force analysis

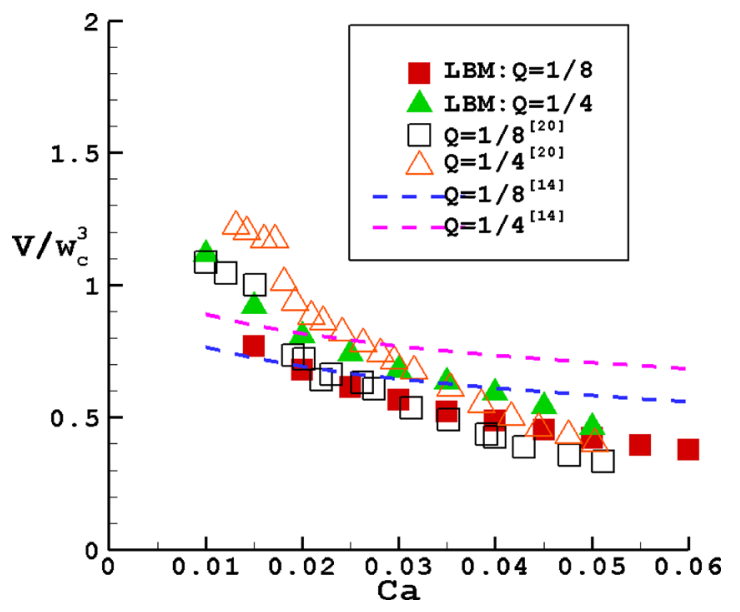

FIG. 3. (Color online) Comparison of normalized volume from the current simulations for $\Lambda=1, \Gamma=1$, viscosity ratio of $\lambda=1 / 8$, and flow ratios $Q=1 / 8$ and $1 / 4$ with the simulations of De Menech et al. (Ref. 20) and the experimental correlation of Christopher et al. (Ref. 14).

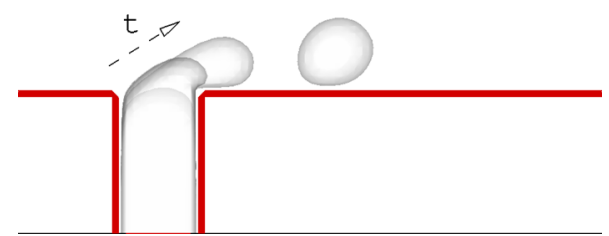

FIG. 4. (Color online) Formation of droplet in the dripping regime $(\mathrm{Ca}=0.1)$ illustrated using superimposed snapshots at different time instants to show the movement of the dispersed phase interface.

was observed, which was also the case in the original experiments, ${ }^{14}$ and could be attributed to the approximations in the form of the forces.

A time history of the movement of the two-fluid interface is shown in Fig. 4 for $\mathrm{Ca}=0.1$ and $\lambda=1 / 10$ with a mesh consisting of $201 \times 41 \times 11$ nodes. In order to check for grid independence, the mesh was refined to $402 \times 82 \times 22$ nodes (i.e., refinement of $100 \%$ ). Since the discretized nondimensional Boltzmann's equation uses a constant value of the lattice speed for the fluid particles to travel from one lattice to the next in a single time step $(c=\Delta x / \Delta t=1)$, a finer spatial mesh implicitly leads to a finer temporal resolution. As a result of this refinement, the droplet radius was found to change by less than $2 \%$, small enough to justify the use of a coarser mesh to save computational time in performing three-dimensional simulations.

For $\mathrm{Ca}>0.01$, as compared to the contribution of the pressure force, the shear action by the continuous phase on the emerging dispersed phase is the dominating mechanism. Figure 5 shows a comparison of droplet shapes for $Q=1 / 20$ and $\lambda=1 / 10$ for a range of capillary numbers with the geometric parameters $\Lambda=0.5$ and $\Gamma=0.33$. The role of the increasing viscous forces on the size of droplets formed at the T-junction is self-evident. For low $\mathrm{Ca}$, the droplets formed are in the form of plugs, with characteristic dimension of the order of the continuous channel width. As the capillary number increases, the "plug" shape changes into "disklike" droplets, a zone which is termed as the transition regime. In this regime, the viscous forces are significant enough to influence the formation of droplets. Beyond a critical capillary number $\left(\mathrm{Ca}_{c}\right)$, the droplets are not confined by the width of the channel and size decreases rapidly, with

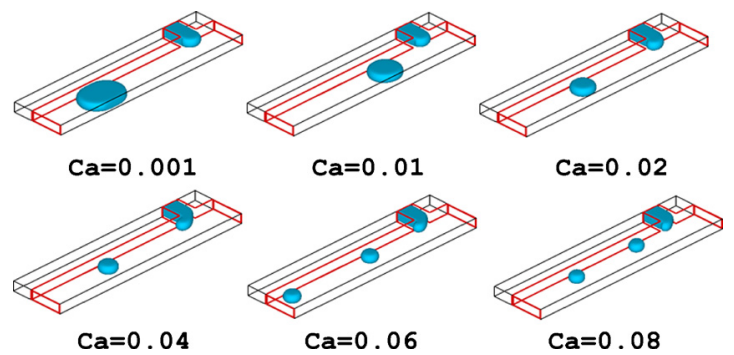

FIG. 5. (Color online) Droplet outcomes for the squeezing $(\mathrm{Ca} \ll 1)$ to dripping regime for a flow ratio of $Q=1 / 20, \Lambda=1 / 2$, and $\Gamma=1 / 3$. As the capillary number increases, the droplets cease to be dependent on the width of the continuous phase channel. 


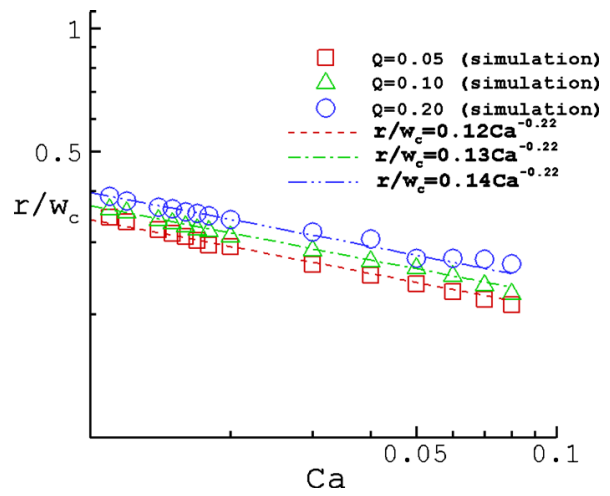

FIG. 6. (Color online) Effective dimensionless radius of the droplets for different flow rate ratios for the geometries $\Lambda=1 / 2$ and $\Gamma=1 / 3$. Symbols represent the simulation results and the dotted lines indicate the curve fits to the numerical data.

shapes that change from disks to that resembling truncated spheres.

The transition regime is also marked with a sharp decrease in the volume of the droplets formed at the T-junction and is also dependent on the viscosity ratio. ${ }^{20}$ Simulations were conducted in the range $0.01 \leq \mathrm{Ca} \leq 0.08$ to capture the effect on droplet shape due to the (relative) competing nature of the pressure and viscous forces for flow rate ratios corresponding to $Q=1 / 5,1 / 10$, and $1 / 20$. In addition, droplet volume and frequency were measured for a viscosity ratio given by $\lambda=1 / 10$ and channel geometric parameters $\Lambda=0.5$ and $\Gamma=0.33$. The droplet volumes were normalized based on the width and depth of the dispersed phase channel, as $\bar{V}=V / V_{0}$, where $V_{0}=w_{d}^{2} h$. The frequency was scaled with a characteristic time $t_{0}=\mu_{d} w_{d} / \sigma$, such that $\bar{f}=f \cdot t_{0}$. The width and viscosity of the dispersed phase have been chosen for defining characteristic scales as these were held constant in the simulations presented herein.

Figure 6 shows the effective normalized radius of the droplets in the transition-to-dripping regime for different dispersed to continuous liquid flow rate ratios. The effective radius is calculated based on an equivalent sphere with the same volume as the droplets formed at the T-junction. Results indicate that the droplet size decreases slowly between $\mathrm{Ca} \in[0.01,0.015]$ and faster thereafter. The critical value of the capillary number $\mathrm{Ca}_{c} \approx 0.015$ is found to be consistent with earlier simulations, ${ }^{20}$ where the critical capillary number depends on the viscosity ratio. ${ }^{12,20}$ In addition, current simulations indicate that the equivalent droplet radius in the transition-to-dripping regime depends on the capillary number and flow rate ratio through the following relationship:

$$
r_{s p h} / w_{c}=0.17 \mathrm{Ca}^{-0.22} Q^{0.11}
$$

for $1 / 20 \leq Q \leq 1 / 5$ and $0.01 \leq \mathrm{Ca} \leq 0.08$. The correlation could not be expanded for higher capillary numbers as the flow regime transformed into a stable jet for any further increase in $\mathrm{Ca}$, an observation that will be further discussed in a later section of this paper. The value of the exponent of $\mathrm{Ca}$ is similar to an earlier observation ${ }^{10}$ [see Eq. (8)], where the exponent was computed to be equal to 0.25 , which further

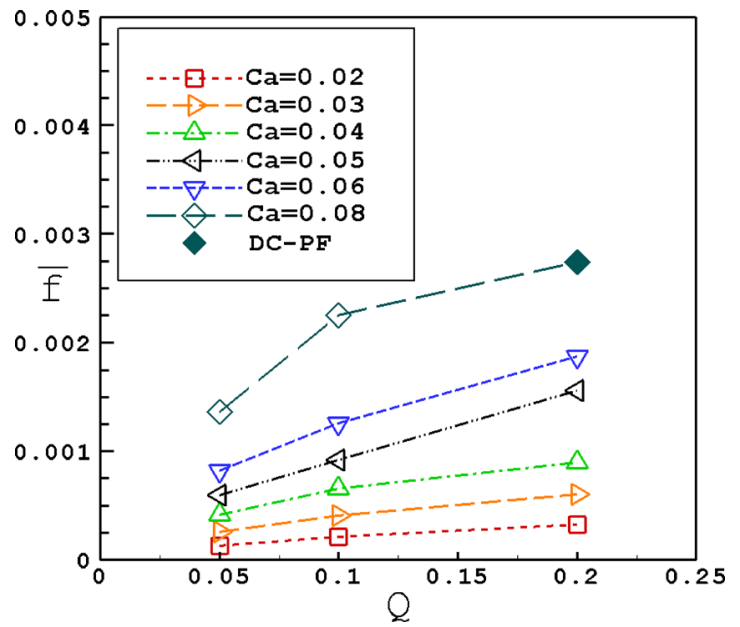

FIG. 7. (Color online) Dimensionless frequency of droplet formation for various capillary numbers in the transition-to-dripping regime for $\lambda=1 / 10$, $\Lambda=1 / 2$, and $\Gamma=1 / 3$.

validates the results of the current lattice Boltzmann simulations.

In addition to droplet size, the frequency of formation was also calculated and is shown in Fig. 7. The capillary number was increased by increasing the flow rate of the continuous liquid, keeping all other physical properties the same. From Fig. 7, it can be observed that for a constant $\mathrm{Ca}$, the frequency of formation of the droplets increases as the flow rate ratio $Q$ increases. Current simulations indicate that increasing $Q$ for a constant $\mathrm{Ca}$ does not translate to an increase in volume and decrease in frequency. Instead, we observed a simultaneous increase in normalized frequency and volume, due to an increase in $Q$, which can also be observed from Eq. (25), where $\bar{V} \propto Q^{0.33}$ and therefore $\bar{f} \propto Q^{0.67}$. For instance, from Fig. 7, it can be observed that doubling the flow rate ratio from $Q=0.05$ to $Q=0.1$ for $\mathrm{Ca}=0.08$ does not lead to a twofold increase in the droplet frequency. From conservation of mass, it is clear that the volume will also increase between these two flow rate ratios. However, for $\mathrm{Ca}=0.08$, the droplet frequency does not increase linearly. This is due to the fact that at $Q=0.2$, droplets are formed in the channel (DC) and the flow appears to be in transition between the DC and PF regimes.

Succeeding the transition regime is the dripping mode, in which droplets are primarily formed by the interplay of viscous and interfacial forces on the expanding dispersed phase. Earlier studies ${ }^{10,20}$ have only focused on the simulation of immiscible liquids with similar viscosities and a microchannel geometry of $\Lambda=1$ and $\Gamma=1$. The influence of liquid viscosities and the channel geometry on the frequency of droplet formation and change in flow regime have not yet been analyzed. In the following sections, the dependence of the droplet volume and formation frequency on the geometry and viscosity ratio for $\mathrm{Ca} \geq 0.1$ is discussed and an analysis on the stability of formation of droplets at high capillary numbers is presented. 


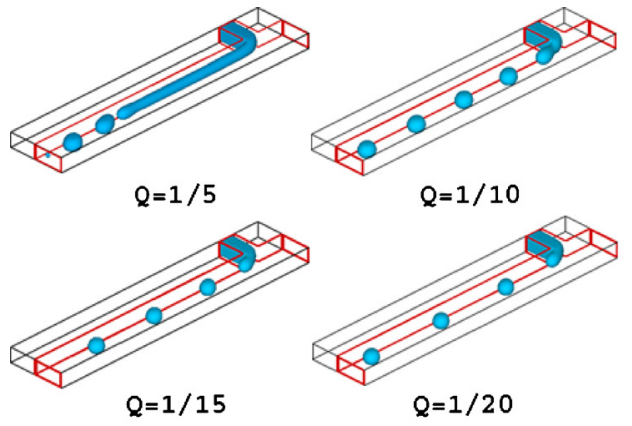

(a) $\Lambda=1 / 2$

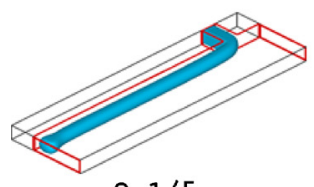

$Q=1 / 5$

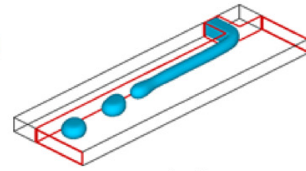

$Q=1 / 10$

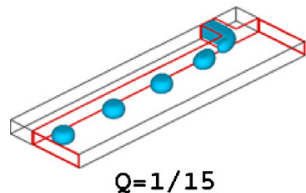

$Q=1 / 15$

(C) $\Lambda=1 / 4$

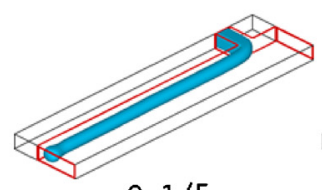

$Q=1 / 5$

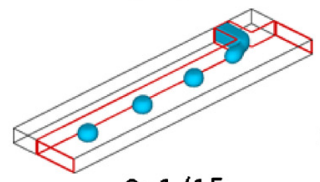

$Q=1 / 15$

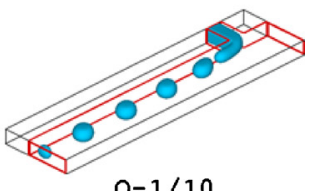

$Q=1 / 10$

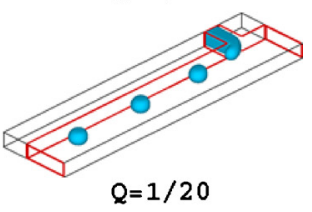

(b) $\Lambda=1 / 3$

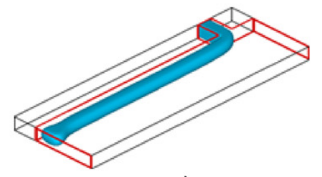

$Q=1 / 5$

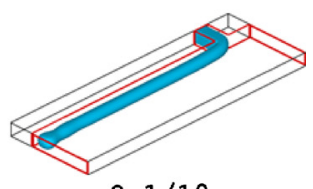

$Q=1 / 10$
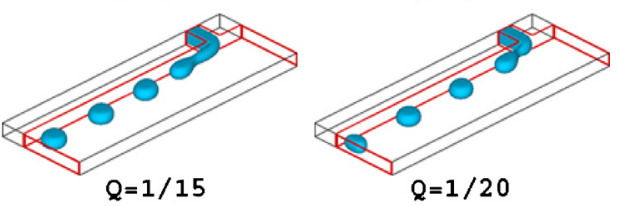

(d) $\Lambda=1 / 5$

FIG. 8. (Color online) Flow regimes for different flow rate ratios and different widths $w_{c}$ for $\mathrm{Ca}=0.1$ and $\lambda=1 / 10$.

\section{A. Effect of geometry}

In the current study, the effect of the width of the continuous phase channel is investigated using fixed dimensionless parameters (namely, $\mathrm{Ca}, Q$, and $\lambda$ ) in the context of the formation of droplets in the dripping regime. Figure 8(a) shows the modes and shapes of droplets formed at $\mathrm{Ca}=0.1$ and $\lambda=\mu_{d} / \mu_{c}=1 / 10$ for $\Lambda=w_{d} / w_{c}=1 / 2$. The droplets formed are near spherical for a low flow rate ratio given by $Q=1 / 20$. As the flow rate ratio is gradually increased by increasing the dispersed phase flow velocity (and keeping the continuous flow rate fixed), the frequency of formation of droplets increased and so does the size. The droplets remained nearly spherical in shape until $Q=1 / 5$. At this value, a thread appeared that penetrates deep into the main channel and droplet formation occurs some distance from the T-junction. Such a situation is commonly known as DC and has been observed earlier. ${ }^{12}$ For higher flow rate ratios, the thread transforms into a stable jet. Simulations were repeated for a range of flow rate ratios and different device geometries by varying the width of the continuous phase channel keeping the capillary number fixed. In this study, the widths have been chosen such that $\Lambda=1 / 2,1 / 3,1 / 4$, and $1 / 5$. Results for $\Lambda=1 / 3$ are shown in Fig. 8(b). For $Q \leq 1 / 15$, spherical droplets are formed at the T-junction. As the dispersed phase flow rate $Q_{d}$ is further increased such that $Q=1 / 10$, the formation of a thread that penetrates into the main channel is evident and droplets similar to the shape of a truncated sphere are formed, as shown in Fig. 8(b). At higher $Q_{d}$, the dispersed phase forms a stable jet that remains parallel to the continuous outer liquid. For higher $w_{c}$, a similar observation can be made. In Fig. 8(c), droplet formation for $\Lambda=1 / 4$ for
$\mathrm{Ca}=0.1$ is shown. For the range of flow rate ratios studied, the droplets formed are found to be nonspherical and highly confined due to the finite depth of the microfluidic assembly. More so, the thread formed at $Q=1 / 10$ penetrates deeper into the main channel and turns into a jet for higher flow rate ratios. For $\Lambda=1 / 5$, as shown in Fig. 8(d), the thread appears at $Q=1 / 15$ and transforms in to stable jet at $Q=1 / 10$.

Repeating the procedure for higher capillary numbers and a range of flow rate ratios for each of the four channel geometries $(\Lambda=1 / 2,1 / 3,1 / 4$, and $1 / 5)$ revealed a similar behavior. However, quantitative differences exist: (a) the droplet size increases with an increase in $w_{c}$ (i.e., decreasing $\Lambda$ ) and (b) for a fixed $\Lambda$, as the capillary number is increased, the width of the zone DC decreases. Intuitively, the increase in the width of the continuous phase channel would result in a smaller gradient of velocity near the dispersed phase channel inlet, which leads to a larger amount of dispersed liquid to expand in the main channel. For higher flow rates of the dispersed phase (i.e., increasing $Q$ ), the shear force to break the liquid stream into droplets may be insufficient as well. The results shown in Fig. 8 corroborate the same.

In Fig. 9, the demarcations that separate the three flow regimes are shown. Based on data collected through numerical simulations, the demarcations that predict the critical flow rate ratio where the transitions from DTJ to DC occur can be identified based on the dependence given by

$$
Q=0.048 \Lambda^{1.095} \mathrm{Ca}^{-0.718}
$$

and for the DC-PF transition

$$
Q=0.055 \Lambda^{0.973} \mathrm{Ca}^{-0.884} \text {. }
$$




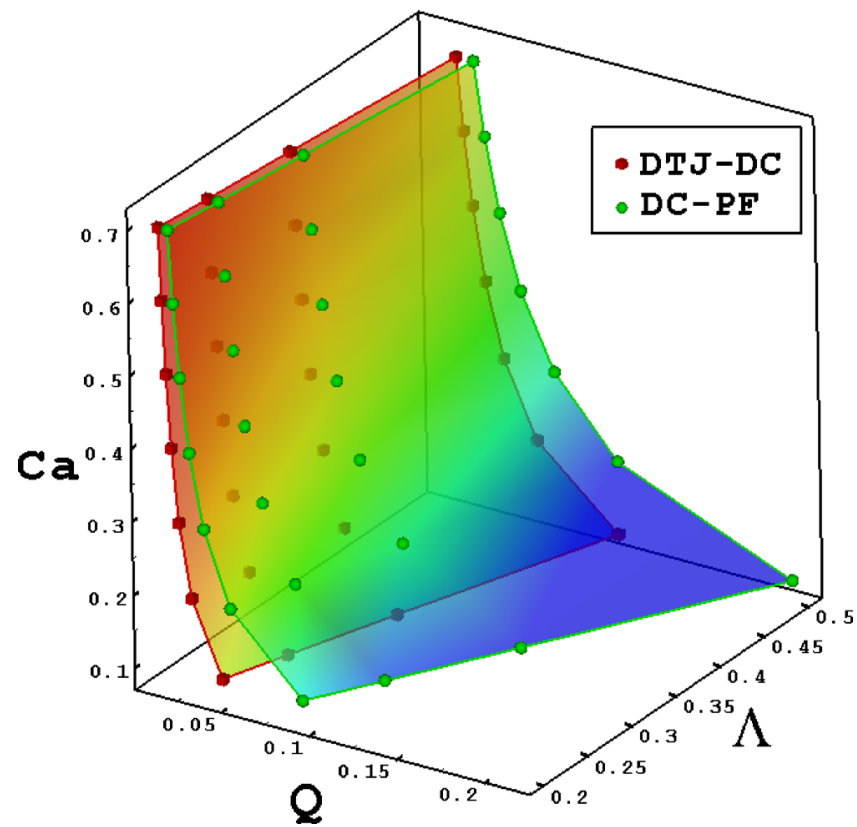

FIG. 9. (Color online) Droplet flow regimes as a function of flow rate ratio (q) and capillary number (Ca) at viscosity ratio $\lambda=1 / 10$ for different inlet widths $(1 / 5 \leq \Lambda \leq 1 / 2)$ of the continuous liquid channel. The boundary between DTJ-DC and DC-PF is given by Eq. (27).

In Eq. (26), the coefficient and exponents were chosen based on a regression analysis. Comparing the widths of the DC regime for two different channels corresponding to $\Lambda=1 / 2$ and $\Lambda=1 / 5$ reveals that this zone becomes thinner as the continuous channel width increases. In addition, transition from DTJ to DC (and DC to PF) occurs at much lower flow ratios for a lower $\Lambda$ (i.e., higher $w_{c}$ ). Moreover, at high $\mathrm{Ca}(\mathrm{Ca} \geq 1)$, the droplet formation may directly transition from DTJ to PF.

The variation of droplet formation frequency $(\bar{f})$ and volume $(\bar{V})$ as a function of the width ratio $(\Lambda)$ is shown in Fig. 10 . For a fixed flow rate ratio $Q$, the nondimensional frequency decreases only marginally with an increase in the

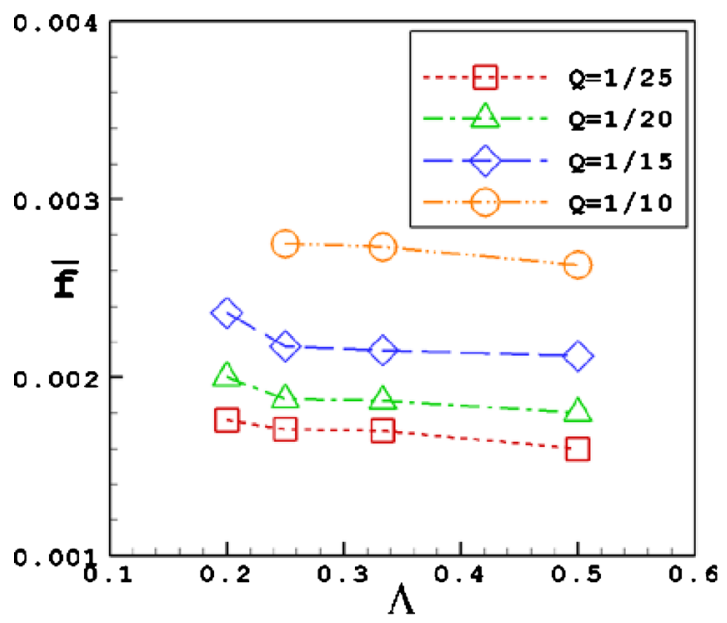

(a) width ratio. Thus, for a fixed $\mathrm{Ca}$, the frequency at which droplets may be collected downstream is not sensitive to the continuous channel width $w_{c}$. In addition, for a fixed $\Lambda\left(=w_{d} / w_{c}\right)$, frequency increases with an increase in $Q$. As the frequency has a weak dependence on the geometry of the device, it can be expected that the volume will increase at a higher rate with the width of the continuous phase channel (or decreasing $\Lambda$ ). Note that based on the characteristic volume and frequency scales and through mass conservation, the dimensionless frequency and volume can be correlated as

$$
\bar{f} \cdot \bar{V}=\frac{\lambda}{\Lambda} Q \mathrm{Ca} .
$$

Thus, for a constant frequency, the volume can be expected to decrease as $\Lambda^{-1}$. This fact is also corroborated from the results shown in Fig. 10(b), where droplet size increases with an increase in $w_{c}$. Therefore, by increasing the width of the continuous channel for a fixed $\mathrm{Ca}$ and $Q$, much can be gained in terms of an increase in droplet volume at nearly the same frequency.

To identify the relationship between frequency and geometry of the device, simulation measurements for $1 / 5 \leq \Lambda$ $\leq 1 / 2$ and $0.1 \leq \mathrm{Ca} \leq 0.7$ were used to construct an empirical correlation to predict the frequency of formation of droplets in the DTJ regime. The frequency was found to vary with the capillary number, flow rate ratio, and the channel width ratio as

$$
\bar{f}=k_{f} \Lambda^{-a} \mathrm{Ca}^{b} Q^{c}
$$

where $k_{f}=0.636, a=0.057, b=1.683$, and $c=0.575$. Since the frequency calculations for only droplets formed in the DTJ regime were used, the flow rate ratio is limited by the prediction of Eq. (26) with the parameters for the DTJ-DC regime. Using Eqs. (26) and (28), the normalized volume of droplets formed in the DTJ regime could be easily estimated.

The frequency (and volume) calculations reported in this study were examined for a different set of flow conditions. Keeping $\mathrm{Ca}=0.1, \lambda=1 / 10$, and fixed $Q_{d}$ and $U_{c}$, the fre-

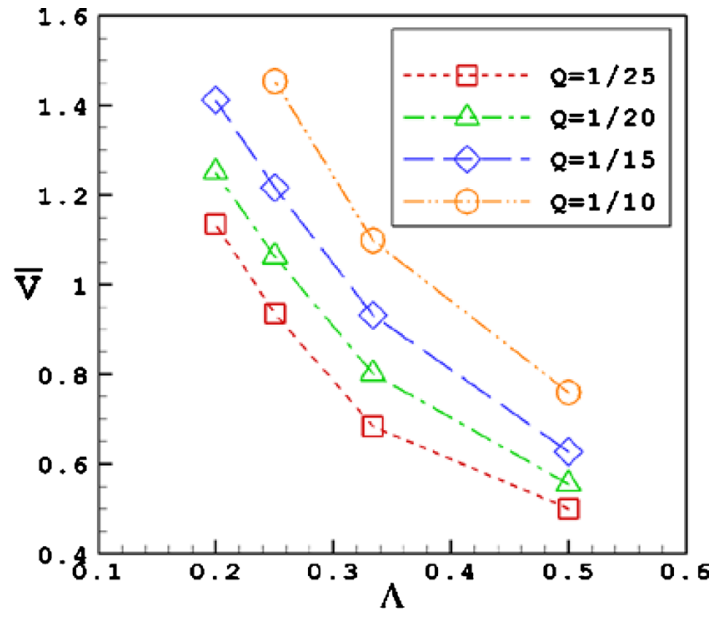

(b)

FIG. 10. (Color online) Variation of dimensionless (a) frequency and (b) volume as a function of width ratio for different flow rate ratios at $\mathrm{Ca}=0.1$ and $\lambda=1 / 10$. 

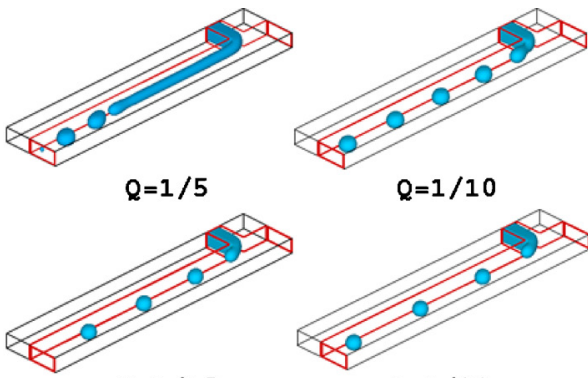

$Q=1 / 15$

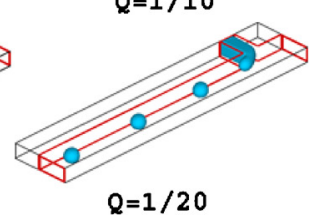

(a) $\lambda=1 / 10$
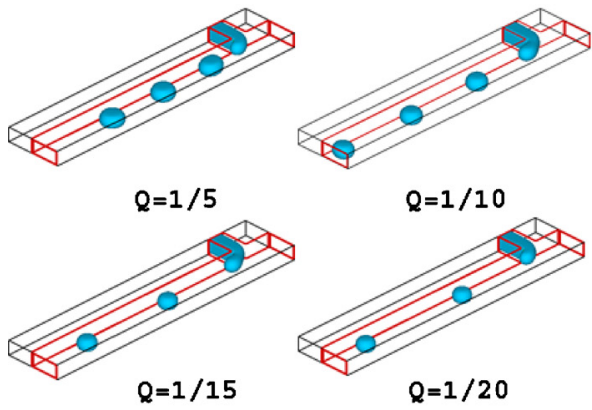

(c) $\lambda=1 / 50$

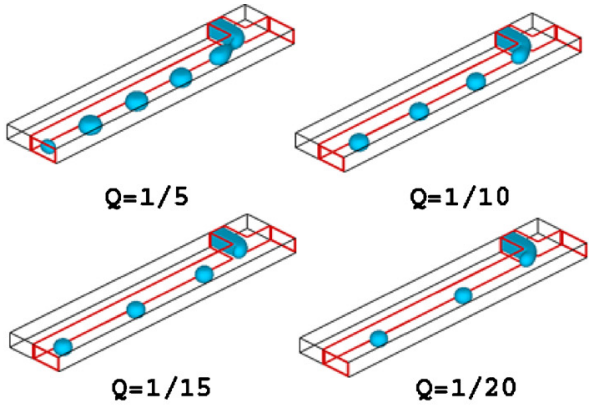

(b) $\lambda=1 / 30$

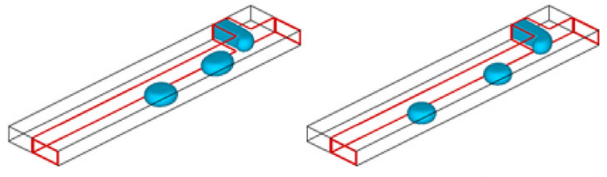

$Q=1 / 5$

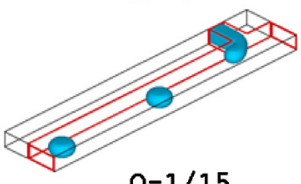

$Q=1 / 15$

(d) $\lambda=1 / 100$

FIG. 11. (Color online) Droplet formation for $\mathrm{Ca}=0.1$ and $\Lambda=1 / 2$ and different viscosity ratios.

quency and volume measurements were compared for two different geometries corresponding to $\Lambda=1 / 2$ and $\Lambda=1 / 4$. The volume was found to increase as $w_{c}$ was increased; for $\Lambda=1 / 2$ and $\bar{V}=0.195$ and for $\Lambda=1 / 4$ and $\bar{V}=1.11$. The dimensionless frequency recorded decreased from 5.135 $\times 10^{-3}$ to $1.803 \times 10^{-3}$, respectively. These results show that for a fixed $\mathrm{Ca}$ and $\lambda$, the droplet volume has an inverse dependence with $w_{c}$, which is consistent with earlier findings. ${ }^{2,14}$ However, the results presented in Fig. 10 correspond to constant $\mathrm{Ca}, Q$, and $\lambda$. Thus, a distinction needs to be made before interpreting the nature of these results.

\section{B. Effect of viscosity}

The critical capillary number at which the transition from droplet to parallel flow occurs is a function of the viscosity ratio. ${ }^{2} \mathrm{~A}$ high viscosity ratio (i.e., small $\mu_{\mathrm{c}}$ ) indicates a relatively smaller shear force on the penetrating dispersed phase and hence a higher likelihood that the thread will remain stable and form a parallel jet. In the current work, the effect of viscosity has been studied in the context of flow regimes that emanate for different viscosities of the continuous phase. For the sake of comparison, the capillary number is held fixed at $\mathrm{Ca}=0.1$ and the geometric parameters are fixed at $\Lambda=1 / 2$ and $\Gamma=1 / 2$. The only parameters varied are $\lambda$ and $Q$.

Droplet regimes for $\lambda=1 / 10,1 / 30,1 / 50$, and $1 / 100$ are shown in Figs. 11(a)-11(d). Based on these results, a common theme emerges. The size of the droplets increases as the flow rate ratio $Q$ increases. For a fixed $Q$, the droplets formed become more confined and resemble truncated spheres as $\mu_{c}$ increases. More so, for the highest simulated flow rate ratio of $Q=1 / 5$, the formation of droplets is facilitated by increasing $\mu_{c}$ as the dispersed phase transforms from PF to DC to DTJ. This indicates that the droplet size and frequency could be controlled by suitable addition of surfactants that may alter the viscosity of the continuous liquid.

The effect of viscosity ratio $\lambda$ on frequency of droplet formation and volume for different flow rate ratios $Q$ at $\mathrm{Ca}=0.1$ and $\Lambda=1 / 2$ is shown in Fig. 12. As can be observed, the frequency increases with an increase in the viscosity ratio $\lambda$ (or decreasing $\mu_{c}$ ). For $Q=1 / 5$, any increase in $\lambda$ beyond $\mu_{c}=30 \mu_{d}$ resulted in droplet formation pushed into the channel (DC); hence that data point is not plotted in Fig. 12. For a fixed $\lambda$, the frequency of droplet formation increases as the flow rate ratio increases. Although the trend is clear on the influence of viscosity ratio, not enough points could be obtained in DTJ to obtain a correlation.

The results of Fig. 12 indicate that the frequency is strongly dependent on the viscosity ratio in the range chosen in this study. From the log-log plot of Fig. 12(a), it is clear that the frequency varies as $\bar{f} \sim \lambda^{1+\eta}$, where $0<\eta<1$. Analytically, the right hand side of Eq. (27) is a linear function of viscosity ratio for a constant value of the other parameters. Therefore, the volume of the formed droplets should scale as $\bar{V} \sim \lambda^{-\eta}$. The variation of droplet volume with viscosity ratio, as shown in Fig. 12(b) indicates the same trend. Moreover, since $\bar{V} \sim \lambda^{-\eta}$, the droplet volume decreases rapidly for small $\lambda$ and gradually thereafter. Thus, for a fixed $\mathrm{Ca}$ and $Q$, the droplet formation frequency (volume) can be increased (decreased) by lowering the continuous phase viscosity (and thus increasing the viscosity ratio). 


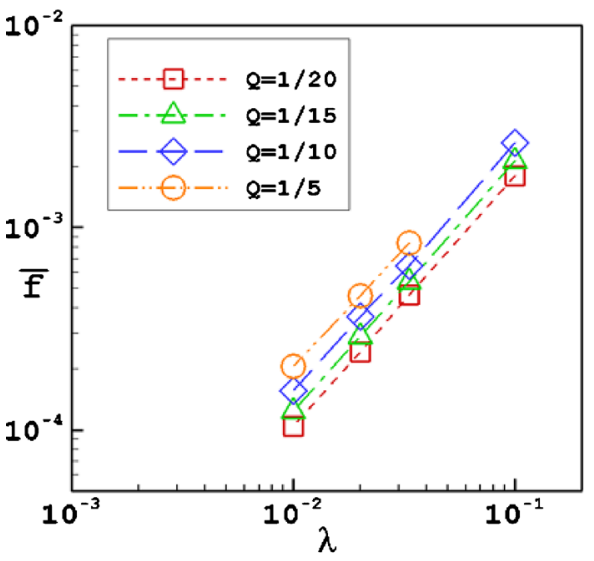

(a)

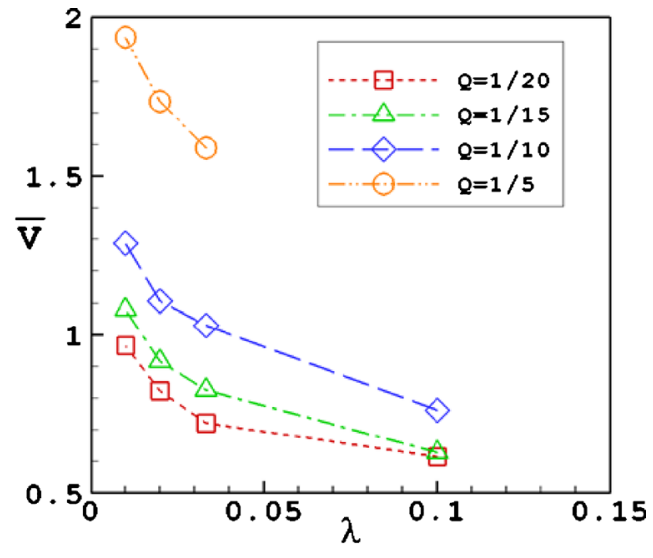

(b)

FIG. 12. (Color online) Variation of dimensionless (a) frequency and (b) volume as a function of viscosity ratio for different flow rate ratios at Ca=0.1 and $\Lambda=1 / 2$.

The increase in frequency with a decrease in continuous phase viscosity can be explained as follows. In the parametric study conducted in this work, the focus is on varying a single dimensionless parameter while keeping others fixed. Thus, increasing $\mu_{c}$ for fixed $\mathrm{Ca}, \Lambda$, and $Q$ leads to a simultaneous decrease in both $U_{c}$ and the dispersed phase flow rate $Q_{d}\left(\because Q_{d}=Q U_{c} w_{c}\right)$. This leads to a lowering in the drag on the penetrating dispersed liquid (as $\operatorname{Drag} \propto C_{D} U_{c}^{2}$, where $C_{D}$ is the drag coefficient ${ }^{21}$ and hence a longer residence time of the dispersed phase in the main channel, as well as a higher volumetric flow of the dispersed phase into the main channel. Therefore, droplets with a relatively higher volume (and lower frequency) are formed. The increase in volume (and decrease in frequency) with increasing $\mu_{c}$ is also consistent with earlier simulations. ${ }^{20,21}$

The dependence of frequency on continuous phase Reynolds number and flow rate was also investigated. As shown in Fig. 13, the dimensionless frequency increases as the continuous phase Reynolds number increases. For a fixed flow rate ratio $Q$, if $Q_{c}$ increases, the shear on the penetrating droplet would also be higher and hence the frequency of formation would increase.

A distinction needs to be made in inferring the results of this section. It is expected that the frequency of formation, for fixed flow rates of the two liquids (i.e., $Q_{c}=$ constant and $Q_{d}=$ constant) and for the same geometry, should increase if the viscosity of the continuous phase is increased, which may seem contrary to results presented in Fig. 12. To verify this, a comparison was made between two viscosity ratios corresponding to $\lambda=1 / 10$ and $\lambda=1 / 50$, where the ratio was changed by increasing $\mu_{c}$. The geometry was fixed $(\Lambda=1 / 2)$ and the continuous and dispersed phase flow rates $\left(Q_{c}\right.$ and $\left.Q_{d}\right)$ were also held constant for the two cases (thus $Q=$ constant). The volume of the drops was found to decrease from $\bar{V}=0.289$ to $\bar{V}=0.182$ as the continuous phase viscosity was increased from $\mu_{c}=10 \mu_{d}$ to $\mu_{c}=50 \mu_{d}$; correspondingly, the frequency increased from $\bar{f}=3.465 \times 10^{-3}$ to $\bar{f}=5.507 \times 10^{-3}$, respectively. Thus, the frequency of droplet formation increases as $\lambda$ decreases [and $\mathrm{Ca}\left(=\mu_{c} U_{c} / \sigma\right)$ increases], as opposed to the selection of variables presented in Fig. 12 where the frequency is shown to increase with $\lambda$ when $\mathrm{Ca}, \Lambda$, and $Q$ are held fixed.

\section{CONCLUSIONS}

In this study, a parametric analysis of droplet formation at T-junction microchannels has been presented. Flow regimes obtained as a result of two immiscible liquids interacting at a T-junction device have been analyzed for transitional to high capillary numbers $(\mathrm{Ca})$, different flow rate ratios $(Q)$, varying width ratios of the dispersed and continuous phase channel $(\Lambda)$, and a wide range of dispersed to continuous liquid viscosities $(\lambda)$. Unlike the low capillary number regime, where the formation of droplets occurs due to an interplay between pressure and interfacial forces, ${ }^{9,20,22}$ these high capillary number flows have been observed to be influenced by the strength of the viscous and interfacial forces acting on the emerging dispersed liquid. Through three-

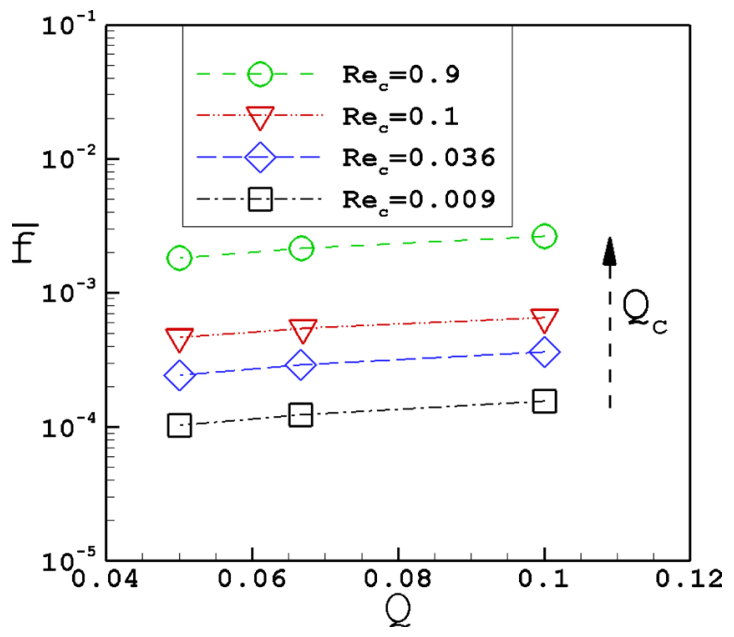

FIG. 13. (Color online) Dimensionless frequency as a function of flow rate ratio for different flow rates of the continuous fluid at $\mathrm{Ca}=0.1$ and $\Lambda=1 / 2$. 
dimensional lattice Boltzmann simulations, it was shown that (for $\lambda=1 / 10, \Lambda=0.5$, and $\Gamma=0.33$ ) in the range $1 / 20 \leq Q$ $\leq 1 / 5$ and $0.01 \leq \mathrm{Ca} \leq 0.08$ the droplet radius can be described by $r_{s p h} / w_{c}=0.17 \mathrm{Ca}^{-0.22} Q^{0.11}$.

In the dripping regime, increasing the flow rate of the dispersed phase can lead to a shift in the droplet formation pattern. For a range of continuous channel widths it was shown that increasing the flow rate ratio results in droplet formation to not occur at the T-junction. Instead, the dispersed phase penetrates the main channel forming a stable thread. The thread transforms into a stable jet for higher flow rates of the dispersed phase. Increasing the width of the channel leads to the transition from DTJ to DC and DC to PF at lower flow rate ratios, as well as the reduction in the width of the intermediate (i.e., DC) zone. The critical flow rate ratio where the transition of the flow patterns occurs was found to obey the relationship $Q=Q_{0} \Lambda^{m} \mathrm{Ca}^{-n}$, where $Q_{0}, m$, and $n$ are constant parameters determined for the range given by $1 / 5 \leq \Lambda \leq 1 / 2$ and $0.1 \leq \mathrm{Ca} \leq 0.7$. For this range of channel geometries and capillary numbers, a correlation to predict the frequency of droplets formed in the DTJ regime was constructed. The frequency was found to vary with the capillary number, flow rate ratio, and the channel width ratio as $\bar{f}=k_{f} \Lambda^{-a} \mathrm{Ca}^{b} Q^{c}$. For a fixed capillary number and flow rate ratio, the frequency of droplet formation was weakly affected by the continuous channel width. As a result, larger droplets were formed by increasing the width of the continuous phase channel.

The effect of continuous phase viscosity was also analyzed for the different flow regimes. For a constant capillary number, channel widths ratio, and flow rate ratio, the droplet frequency increased as the continuous phase viscosity was decreased. Moreover, the droplet volume increased with an increase in the continuous phase viscosity. Thus, to achieve a higher rate of formation of droplets in a practical scenario, decreasing the continuous phase viscosity, and not the channel width, should be the preferred choice. However, changing the continuous channel width would be beneficial if the objective is to manipulate the volume of droplets at a fixed frequency.

In addition, a tenfold increase in viscosity allowed the formation of droplets even at higher flow rates. These important results suggest that the process of droplet formation can be controlled by suitably altering the continuous phase viscosity.

${ }^{1}$ H. A. Stone, A. D. Stroock, and A. Ajdari, "Engineering flows in small devices: Microfluidics toward a lab-on-a-chip," Annu. Rev. Fluid Mech. 36, 381 (2004).

${ }^{2}$ G. F. Christopher and S. L. Anna, "Microfluidic methods for generating droplet streams," J. Phys. D: Appl. Phys. 40, R319 (2007).

${ }^{3}$ P. B. Umbanhowar, V. Prasad, and D. A. Weitz, "Monodisperse emulsion generation via drop break off in a coflowing stream," Langmuir 16, 347 (2000).

${ }^{4}$ C. Cramer, P. Fischer, and E. J. Windhab, "Drop formation in a co-flowing ambient fluid," Chem. Eng. Sci. 59, 3045 (2004).

${ }^{5}$ S. L. Anna, N. Bontouz, and H. A. Stone, "Formation of dispersions using 'flow focusing' in microchannels," Appl. Phys. Lett. 82, 364 (2003).
${ }^{6}$ P. Garstecki, M. J. Fuerstman, and G. M. Whitesides, "Nonlinear dynamics of a flow-focusing bubble generator: An inverted dripping faucet," Phys. Rev. Lett. 94, 234502 (2005).

${ }^{7}$ Y.-C. Tan, J. S. Fisher, A. I. Lee, V. Cristini, and A. P. Lee, "Design of microfluidic channel geometries for the control of droplet volume, chemical concentration, and sorting," Lab Chip 4, 292 (2004).

${ }^{8}$ T. Thorsen, R. W. Roberts, F. H. Arnold, and S. R. Quake, "Dynamic pattern formation in a vesicle-generating microfluidic device," Phys. Rev. Lett. 86, 4163 (2001).

${ }^{9}$ P. Garstecki, M. J. Fuerstman, H. A. Stone, and G. M. Whitesides, "Formation of droplets and bubbles in a microfluidic T-junction-Scaling and mechanism of break-up," Lab Chip 6, 437 (2006).

${ }^{10}$ S. Van der Graaf, T. Nisisako, C. G. P. H. Schroën, R. G. M. van der Sman, and R. M. Boom, "Lattice Boltzmann simulations of droplet formation in a T-shaped microchannel," Langmuir 22, 4144 (2006).

${ }^{11}$ J. D. Tice, A. D. Lyon, and R. F. Ismagilov, "Effects of viscosity on droplet formation and mixing in microfluidic channels," Anal. Chim. Acta 507, 73 (2004).

${ }^{12} \mathrm{P}$. Guillot and A. Colin, "Stability of parallel flows in a microchannel after a T-junction," Phys. Rev. E 72, 066301 (2005).

${ }^{13}$ J. Husny and J. J. Cooper-White, "The effect of elasticity on drop creation in T-shaped microchannels," J. Non-Newtonian Fluid Mech. 137, 121 (2006).

${ }^{14}$ G. F. Christopher, N. N. Noharuddin, J. A. Taylor, and S. L. Anna, "Experimental observations of the squeezing-to-dripping transition in T-shaped microfluidic junctions," Phys. Rev. E 78, 036317 (2008).

${ }^{15}$ G. Cristobal, J.-P. Benoit, M. Joanicot, and A. Ajdari, "Microfluidic bypass for efficient regulation of droplet traffic at a junction," Appl. Phys. Lett. 89, 034104 (2006).

${ }^{16}$ J. H. Xu, S. W. Li, G. G. Chen, and G. S. Luo, "Formation of monodisperse microbubbles in a microfluidic device," AIChE J. 52, 2254 (2006).

${ }^{17}$ J. H. Xu, S. W. Li, J. Tan, Y. J. Wang, and G. S. Luo, "Preparation of highly monodisperse droplet in a T-junction microfluidic device," AIChE J. 52, 3005 (2006).

${ }^{18}$ W. Engl, M. Roche, A. Colin, and P. Panizza, "Droplet traffic at a simple junction at low capillary numbers," Phys. Rev. Lett. 95, 208304 (2005).

${ }^{19}$ S. M. S. Murshed, S. H. Tan, and N. T. Nguyen, "Temperature dependence of interfacial properties and viscosity of nanofluids for droplet-based microfluidics," J. Phys. D: Appl. Phys. 41, 085502 (2008).

${ }^{20}$ M. D. Menech, P. Garstecki, F. Jousse, and H. A. Stone, "Transition from squeezing to dripping in a microfluidic T-shaped junction," J. Fluid Mech. 595, 141 (2008)

${ }^{21}$ A. Gupta, S. M. S. Murshed, and R. Kumar, "Droplet formation and stability of flows in a microfluidic T-junction," Appl. Phys. Lett. 94, 164107 (2009).

${ }^{22}$ A. Gupta and R. Kumar, "Effect of geometry on droplet formation in the squeezing regime in a microfluidic T-junction," Microfluid. Nanofluid. 8, 799 (2010).

${ }^{23}$ S. Chen and G. D. Doolen, "Lattice Boltzmann method for fluid flows," Annu. Rev. Fluid Mech. 30, 329 (1998).

${ }^{24}$ A. K. Gunstensen, D. H. Rothman, S. Zaleski, and G. Zanetti, "Lattice Boltzmann model of immiscible fluids," Phys. Rev. A 43, 4320 (1991).

${ }^{25} \mathrm{X}$. Shan and H. Chen, "Lattice Boltzmann model for simulating flows with multiple phases and components," Phys. Rev. E 47, 1815 (1993).

${ }^{26} \mathrm{M}$. Swift, W. Osborne, and J. Yeomans, "Lattice Boltzmann simulation of nonideal fluids," Phys. Rev. Lett. 75, 830 (1995).

${ }^{27}$ T. Inamuro, S. Tajima, and F. Ogino, "Lattice Boltzmann simulation of droplet collision dynamics," Int. J. Heat Mass Transfer 47, 4649 (2004).

${ }^{28}$ S. V. Lishchuk, C. M. Care, and I. Halliday, "Lattice Boltzmann algorithm for surface tension with greatly reduced microcurrents," Phys. Rev. E 67, 036701 (2003)

${ }^{29} \mathrm{X}$. He and L. S. Luo, "Lattice Boltzmann model for the incompressible Navier-Stokes equation," J. Stat. Phys. 88, 927 (1997).

${ }^{30}$ M. Latva-Kokko and D. H. Rothman, "Diffusion properties of gradientbased lattice Boltzmann models of immiscible fluids," Phys. Rev. E 71, 056702 (2005)

${ }^{31}$ S. Succi, The Lattice Boltzmann Equation for Fluid Dynamics and Beyond (Oxford University Press, New York, 2001).

${ }^{32}$ M. M. Dupin, I. Halliday, and C. M. Care, "Simulation of a microfluidic flow-focusing device," Phys. Rev. E 73, 055701(R) (2006). 